\title{
Charge recombination in soft $x$-ray laser produced nanoplasmas
}

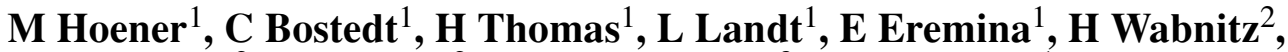 \\ T Laarmann $^{2}$, R Treusch ${ }^{2}$, A R B de Castro ${ }^{3}$ and T Möller ${ }^{1}$ \\ ${ }^{1}$ Institut für Optik und Atomare Physik, Technische Universität Berlin, Eugene-Wigner-Bldg EW 3-1, \\ Hardenbergstr 36, 10623 Berlin, Germany \\ ${ }^{2}$ HASYLAB, DESY, 22603 Hamburg, Germany \\ ${ }^{3}$ LNLS and Universidade Estadual de Campinas, Campinas SP, Brazil \\ E-mail: bostedt@physik.tu-berlin.de
}

Received 12 August 2008

Published 2 September 2008

Online at stacks.iop.org/JPhysB/41/181001

\begin{abstract}
The ionization and charge separation processes of nanoplasmas created by resonant excitation of atomic clusters in intense soft x-ray pulses have been investigated. Through irradiation with femtosecond pulses from the FLASH free electron laser (FEL) at $\lambda=13.7 \mathrm{~nm}$ and power densities exceeding $10^{14} \mathrm{~W} \mathrm{~cm}^{-2}$ the clusters are highly ionized with transient atomic charge states up to $9+$. Variation of the cluster composition from pristine to doped and core-shell systems allows tracking of the spatial origin and charge states of the fragments yielding insight into the nanoplasma dynamics. The data give evidence for efficient charge redistribution processes leading to a Coulomb explosion of the cluster outer part and recombination of the nanoplasma core. The experiments show qualitatively different processes for (soft) x-ray produced nanoplasmas from the optical (IR) strong-field regime where the clusters disintegrate completely in a Coulomb explosion.
\end{abstract}

(Some figures in this article are in colour only in the electronic version)

Understanding the interaction of light with matter has been a central theme of physics over the past century, starting with the concept of the photon and the inception of quantum theory. The invention of the laser and the continuing advance in laser technologies has made it possible to explore regimes of nonlinear light-matter interaction leading to novel laserbased concepts for particle accelerators, plasma formation and nuclear fusion [1]. Currently, we are witnessing the advent of intense lasers in the $x$-ray regime. One of the most exciting prospects of research with $\mathrm{x}$-ray lasers is direct imaging of nonperiodic nanoscale objects, such as biomolecules, nanocrystals, living cells and viruses [2]. Even though it is crucial for the success of the imaging experiments, understanding the interaction of intense x-ray pulses with atomic systems and the underlying dynamics is still in its infancy. To date, virtually all studies about the ionization as well as nuclear dynamics of nanometer-sized structures in intense (soft) x-ray pulses are of theoretical nature [3-6] and no experimental data are available.

For the experimental investigations of matter in intense light pulses atomic clusters are ideal because their size can be tuned from the molecular to the bulk-like regime and there is no energy dissipation into surrounding media. The ionization dynamics of clusters in intense laser pulses depend considerably on the radiation wavelength. In the infrared spectral regime the cluster is ionized by the optical field and the resulting transient nanoplasma is efficiently heated by the external laser field via inverse bremsstrahlung (IBS) and collective effects, leading to a Coulomb explosion of the cluster [7]. Because the ponderomotive energy scales with $\omega^{-2}$ ( $\omega$-laser frequency) and thus, the direct effect of the laser field on the electron movement is small, it was a big surprise when experiments in the vacuum-ultraviolet spectral regime at $100 \mathrm{~nm}$ and intensities up to $10^{13} \mathrm{~W} \mathrm{~cm}^{-2}$ reported unexpectedly high-energy absorption and complete Coulomb 
explosion of clusters [8]. The efficient energy absorption of the clusters is theoretically explained with more realistic potentials for IBS [9], barrier suppression in the ionized cluster [10] and enhanced heating through many-body collisions in a transient strongly coupled nanoplasma [11]. While theoretically IBS is predicted to be the dominant absorption mechanism down to $62 \mathrm{~nm}$ [12] recent photoemission experiments at $\lambda=32 \mathrm{~nm}$ and similar intensities find no evidence for it [13]. Instead, energy deposition is best described by photoabsorption and photoemission which becomes frustrated in the Coulomb field of the charging cluster, suppressing the formation of a nanoplasma for intensities up to $10^{13} \mathrm{~W} \mathrm{~cm}^{-2}$ [13].

In this communication we present first experimental data about intense laser-cluster interaction with resonant excitation in the soft $\mathrm{x}$-ray regime. Pristine $\mathrm{Xe}, \mathrm{Ar}$ and heterogeneous $\mathrm{Xe}-\mathrm{Ar}$ clusters are irradiated at $\lambda=13.7 \mathrm{~nm}$ and power densities exceeding $10^{14} \mathrm{~W} \mathrm{~cm}^{-2}$ leading to nanoplasma formation with transient charge states up to 9+. The ionization dynamics including charge transfer and charge separation processes are followed by a controlled variation of the cluster composition from pristine over low doping level to core-shell systems. Tracking the specific $m / q$ ratios in time-of-flight (tof) spectra yields insight into the spatial origin and charge states of the fragments. Low doping levels in the bulk and at the surface allow the creation of extreme charge states at selected sites in the cluster for investigation of charge transfer processes. By varying the surface layer thickness of coreshell systems the Cluster explosion and charge recombination processes, i.e., evolution of the nanoplasma are probed. The experiments evidence qualitatively different dynamics in $\mathrm{x}$ ray produced plasmas compared with the optical strong-field regime. The data show efficient charge redistribution within the cluster leading to a Coulomb explosion of the outer layers and recombination of the x-ray excited nanoplasma core which is in contradiction to the standard Coulomb explosion model assuming complete disintegration of the cluster. The current findings are of central importance for evolving strategies to delay the Coulomb explosion of bio-molecules with a tamper [6] for future imaging with x-ray lasers [2, 3, 14].

The experiments are performed at the FLASH free electron laser in Hamburg [15]. The FEL beam is focussed with multilayer optics on a beam spot of $d \approx 15 \mu \mathrm{m}$. Prior to focussing the far wings of the beam are cut with apertures. The average pulse energies are $12 \mu \mathrm{J}$ and the pulse durations are $10 \mathrm{fs}$ [15]. All data are acquired in singleshot mode. With the current experimental conditions power densities exceeding $10^{14} \mathrm{~W} \mathrm{~cm}^{-2}$ are obtained as evidenced by the highest observable charge states of atomic Xe [16]. The photon energy is set to $90.5 \mathrm{eV}(\lambda=13.7 \mathrm{~nm})$ in the middle of the broad $\mathrm{Xe} 4 \mathrm{~d}$ giant resonance $(\Delta E=50 \mathrm{eV})$. The photon absorption cross section for the cluster materials is 24.7 Mbarn for Xe compared with 1.4 Mbarn for Ar, translating to about 17 and 1 photons per photoionization cross section $\sigma$, respectively. The charged cluster fragments are detected with a tof spectrometer with an entrance aperture of $500 \times$ $1000 \mu \mathrm{m}$ perpendicular to the beam which is shorter than the Rayleigh length of the focus. In order to avoid secondary ionization events in the tof spectrometer the cluster beam is heavily diluted with a large source-interaction zone distance, double skimmer set-up, resulting in sample densities of less than 50 clusters in the focal volume under the spectrometer aperture. The clusters are prepared by adiabatic expansion of rare gases through a pulsed $100 \mu \mathrm{m}$ conical nozzle with an half-opening angle of $15^{\circ}$ and their sizes are determined with scaling laws for pristine clusters [17, 18]. For doping of the clusters two different techniques are used. Surface doping is achieved through the pick-up technique, where the Ar cluster jet crosses a Xe beam of atoms from a $600 \mu \mathrm{m}$ nozzle about $10 \mathrm{~mm}$ away and a backing pressure of $1.5 \mathrm{mbar}$ [19]. The Ar host clusters are prepared with a stagnation pressure of 28 bar and a nozzle temperature of $300 \mathrm{~K}$. The Ar clusters doped in the interior and the $\mathrm{Xe} / \mathrm{Ar}$ core-shell systems are prepared by co-expansion of premixed gases. At low concentration of $\approx 0.01 \% \mathrm{Xe}$ in $\mathrm{Ar}$ isolated $\mathrm{Xe}$ atoms are embedded in the Ar matrix [19]. At high concentrations exceeding 5-10\% of $\mathrm{Xe}$ in Ar, pure Xe clusters are formed because then Ar acts merely as a seeding gas which is completely evaporated off the growing cluster. In a narrow range between $1-5 \% \mathrm{Xe}$ in $\mathrm{Ar}$ a strong enrichment of the $\mathrm{Xe}$ in the cluster is observed [20-22]. The Xe nucleates in the core of the Ar cluster but not all Ar is evaporated $[21,22]$. This formation of the core-shell structure is due to phase separation resulting from the different cohesive energies and melting points of $\mathrm{Xe}$ and Ar. Thus, the enrichment factor of Xe in the Ar cluster, or in other words, the thickness of the Ar surface layer, depends sensitively on the Xe concentration of the gas premix. For the current experiments a gas premix of $2 \% \mathrm{Xe}$ in $\mathrm{Ar}$ is expanded at 7 bars and nozzle temperatures of $300 \mathrm{~K}$. These parameters have been chosen because they yield the same gas-independent cluster condensation parameter $\Gamma^{*}$ $[17,18]$ as in previous detailed photoelectron spectroscopy investigations about such systems [21]. The corresponding cluster size according to the scaling laws for pristine clusters $[17,18]$ is $\langle N\rangle \approx 400$ with the typical size distribution $\Delta N \approx N$ full-width at half-maximum. Additionally, a larger core-shell system with $\langle N\rangle \approx 4000$ is prepared by expanding the same gas premix at 17 bars. For the current experimental conditions a Xe enrichment factor of about 10 has been measured in different previous investigations [21-23]. Therefore the core-shell structures can be roughly estimated to consist of a Xe core with approximately 1-2 and 3-4 Ar shells on top, respectively.

In figure 1 the tof data converted to $m / q$ spectra of atomic $\mathrm{Xe}$ as well as pristine $\mathrm{Xe}$ and Ar clusters exposed to the FEL pulse are shown. With the given power densities, Xe atoms (the top panel in figure 1) are multiply ionized with charge states up to $9+$ by sequential single- and multi-photon ionization [16]. In the atomic spectra the Xe isotope distribution is well resolved. Compared to previous measurements [16] a complete depletion of $\mathrm{Xe}^{+}$and reduced intensity of the other low-charge states is observed which can be attributed to the cut low-power density far wings of the beam as described above. The spectrum for Xe clusters is significantly different (the middle panel in figure 1). Here, the singly ionized monomers are the dominant signal and also larger $\mathrm{Xe}_{n}^{+}$fragments are present. In the inset the $m / q$ range of the Xe clusters up to 


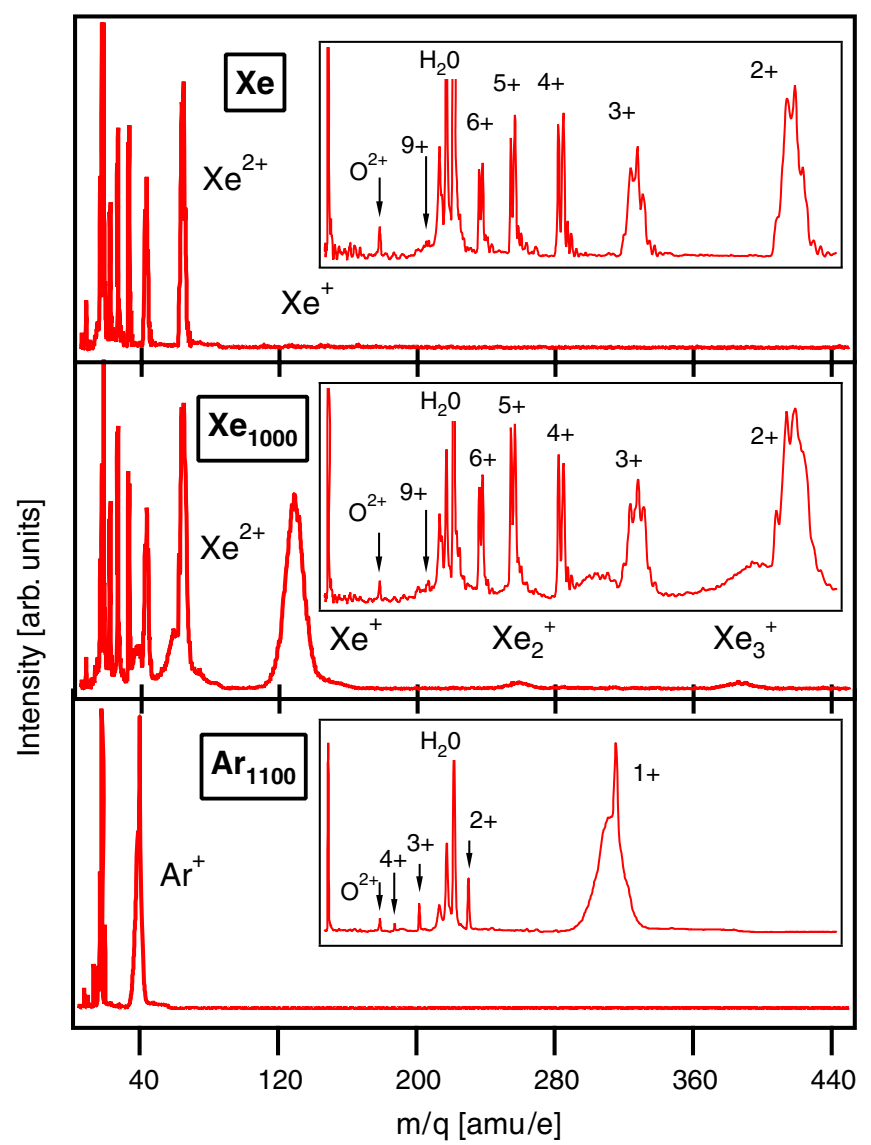

Figure 1. Reference spectra. Top: for Xe atoms highly charged ions up to $\mathrm{Xe}^{9+}$ (inset) are generated during one laser shot. The peak splitting is due to different $\mathrm{Xe}$ isotopes. Middle: $\mathrm{Xe}_{N}$ cluster $(N \sim 1000)$. Larger (dimer, trimer) and multiply charged fragments of the cluster are detected. Bottom: $\operatorname{Ar}_{N}$ clusters $(N \sim 1100)$. The clusters fragment predominantly into monomers. The arrows indicate the peak positions of high charge states without any initial kinetic energies, i.e., atomic contributions.

$70 \mathrm{amu} / \mathrm{e}$ is shown. Here, higher charge states similar to atomic Xe can be identified. Each peak consists of a sharp centre also showing the $\mathrm{Xe}$ isotope distribution which is accompanied by a clear foot towards smaller $m / q$ ratios. The sharp peaks can be attributed to ionization of Xe residual gas whereas the foots are due to the cluster signal. They stem from Xe ions which are accelerated towards the detector during the cluster explosion [8]. Simulations of the spectra indicate that the charged Xe fragments carry on average less than $100 \mathrm{eV}$ kinetic energy. Similarly, for Ar clusters (the bottom panel in figure 1) a broad monomer signal from the cluster explosion dominates the spectra. Higher charge states up to $4+$ can be observed; however, a clear cluster signal fingerprint cannot be identified.

Following the concept of inner and outer ionization of clusters in strong laser fields [7], which is also used for the theoretical descriptions in the short-wavelength regime $[5,10]$, the atoms in the gas jet and the cluster are similarly highly ionized leading to the formation of a highly excited non-thermal nanoplasma [13]. In the optical and vacuumultraviolet $(100 \mathrm{~nm})$ spectral regime the nanoplasma can efficiently absorb a large fraction of the incident laser energy

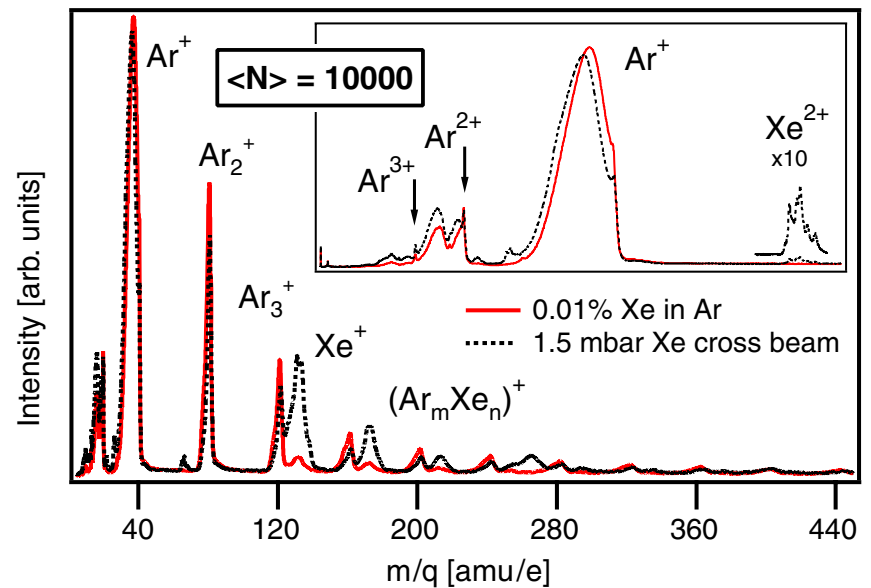

Figure 2. $m / q$ spectra of large Ar clusters with $\langle N\rangle=10^{4}$, doped on the surface by a $1.5 \mathrm{mbar}$ Xe cross beam or in the volume by expansion of a premixed ArXe gas. Pure Ar and Xe fragments as well as mixed ArXe clusters are formed during the explosion.

yielding extreme charge states and high fragment kinetic energies $[1,5,8]$. The data in figure 1 , however, suggest that for clusters no higher charge states than for atoms and only moderate fragment kinetic energies are present. The data show that the cluster dynamics in intense short-wavelength light $(13 \mathrm{~nm})$ differ significantly from the other regimes.

The relaxation and recombination of the created nanoplasma can be followed by investigating doped clusters. Here, the Xe dopant can be considered both a probe to follow site specific charge separation dynamics and a charge supplier to investigate charge transfer processes. The $m / q$ spectra for surface as well as bulk doped Ar clusters are displayed in figure 2. Qualitatively, the heterogenous cluster spectra (figure 2) are similar to those of pristine clusters (figure 1). They are dominated by an intense, broad $\mathrm{Ar}^{+}$monomer peak, but also singly charged larger $\mathrm{Ar}_{n}^{+}$and $\mathrm{Ar}_{m} \mathrm{Xe}_{n}^{+}$fragments as well as clear $\mathrm{Ar}^{2+}$ and $\mathrm{Ar}^{3+}$ cluster signals are present. The dominant $\mathrm{Xe}$ signal for the surface doped clusters (the $1.5 \mathrm{mbar}$ cross beam in figure 2) are the monomer and larger $\mathrm{Ar}_{n} \mathrm{Xe}_{m}$ ionic fragments which are highly stable due to their quasicovalent bonding [24]. Further, no multiply charged Xe from the cluster explosion can be identified. The well-resolved isotope distribution of the weak $\mathrm{Xe}^{2+}$ signal indicates that it does not originate from clusters but from uncondensed atoms in the beam. Similarly, the bulk-doped clusters $(0.01 \% \mathrm{Xe}$ in Ar in figure 2) show a weak $\mathrm{Ar}_{n} \mathrm{Xe}_{m}^{+}$signal and no higher $\mathrm{Xe}$ charge states. Considering that virtually all $\mathrm{Xe}$ atoms are highly charged in the light pulse (figure 1) these results show that there is a very efficient charge transfer process from the $\mathrm{Xe}$ to the surrounding Ar atoms taking place, partly followed by relaxation into molecular fragments. The charge transfer processes occur on a fs-timescale [10], the fragment formation, however, is much slower and on the order of picoseconds [25]. Additionally, the absence of highly charged Xe ions shows that no stabilization and field ionization of high-charge states on the cluster surface is currently happening, which has been speculated about previously $[8,10]$.

Further insight into the charge redistribution and cluster explosion dynamics can be obtained from the core-shell 


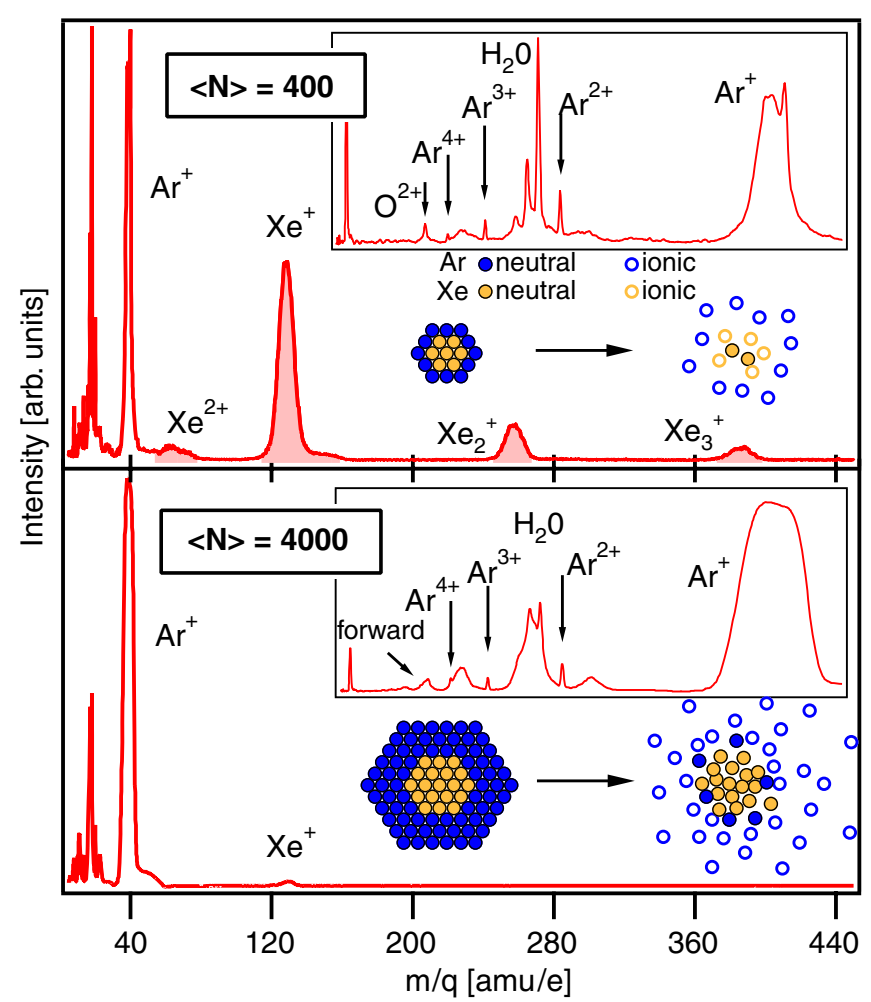

Figure 3. $m / q$ spectrum of Xe core-Ar shell clusters with different sizes. Top: for small clusters $(\langle N\rangle=400)$ predominantly Xe cluster (filled) and Ar fragments are detected. Bottom: for large clusters $(\langle N\rangle=4000)$ the $\mathrm{Xe}$ signal is virtually absent and the Ar signal including highly charged fragments becomes more intense. The insets depict the Xe distribution in the Ar cluster and the proposed expansion mechanism after irradiation. The arrows indicate the peak positions of high charge states without any initial kinetic energies, i.e., atomic contributions.

systems. Here, the $\mathrm{Xe}$ atoms in the core, surrounded by an $\mathrm{Ar}$ shell with variable thickness are resonantly excited to extreme charge states. Such systems are also relevant for future (bio-)molecule tampers [6]. The $m / q$ spectra for the coreshell systems are shown in figure 3. For the smaller systems $(\langle N\rangle \approx 400)$ with a thin Ar coating, the most intense signals stem from Xe fragments, namely $\mathrm{Xe}^{+}, \mathrm{Xe}_{2}^{+}, \mathrm{Xe}_{3}^{+}$and the $\mathrm{Ar}^{+}$ monomer. Further, a small broad signal with $m / q=65$ is present which can be attributed to double charged Xe ions from the cluster. For $m / q<40$ (the inset in figure 3) predominantly higher Ar charge states and contributions from residual gas are present. Each Ar peak consists of a sharp main line from the atomic contribution and a foot on each side stemming from the cluster ions which are accelerated during the Coulomb explosion in or against the direction of the detector [8]. Simulations show that the $\mathrm{Ar}^{3+}$ cluster fragments carry on average $140 \mathrm{eV}$ kinetic energy. Xe ions with charge states higher than two, as seen in the case of atomic Xe and pure Xe clusters (compare figure 1) are not observed. The absence of highly charged Xe ions shows again that a fast charge redistribution from the multiply charged Xe to the surrounding Ar layers takes place. Analyzing the signal intensities in more detail, the ratio of $\mathrm{Xe}$ and Ar (figure 3) is close to one, thus a $\mathrm{Xe}$ enrichment factor of 25 can be deduced which is slightly higher than the factor of 10 reported previously for similar expansion conditions [22]. The spectra of the large Xe core-Ar shell clusters $(\langle N\rangle \approx 4000)$ with a thick Ar coating of about three or more shells look completely different (figure 3 , bottom). The Xe signal is only $0.6 \%$ of the total signal, or in other words virtually absent. The dominant signal is now the Ar monomer. Further, the multiply charged cluster Ar fragment signal becomes much more intense when compared with the smaller core-shell system (upper panel) and the pristine Ar clusters (figure 1). The forward/backward accelerated cluster ions start to override the residual gas peaks. Additionally, forward accelerated quadruply charged ions from the cluster explosion become clearly visible on the left side of the $\mathrm{Ar}^{4+}$ atomic peak. Simulations show that the cluster fragment kinetic energies are for the $\mathrm{Ar}^{3+}$ peak $150 \mathrm{eV}$ and the $\mathrm{Ar}^{4+}$ peak $250 \mathrm{eV}$ and thus, significantly higher than for the pristine Ar clusters. The fact that only a very small amount of $\mathrm{Xe}^{+}$fragments can be detected is surprising because the fraction of $\mathrm{Xe}$ atoms in the cluster is much larger. Following the concept of inner and outer ionization [7], also holding for the short-wavelength regime [5], the Xe atoms in the core become multiply ionized through the FEL pulse. Therefore the strong decrease in the Xe signal and increase in Ar charge states and fragment kinetic energies for larger clusters must be caused by charge transfer, relaxation and separation dynamics, which are related to the size of the cluster and its shell structure. Considering the structure of the clusters, the detected Ar ions can only be ejected from the few outermost Ar surface layers, giving evidence of the Coulomb explosion of the cluster outer part only. The absence of the Xe signal in the tof spectrum (figure 3 , bottom) suggests that the highly charged Xe nanoplasma core recombines with quasifree electrons and thus cannot be detected. The recombination of the nanoplasma core can be understood if the details of the cluster ionization are considered. At short wavelengths and the current power densities, the cluster ionization is best described with direct photoemission in the building Coulomb potential [13]. Since the total energy needed to remove $N$ electrons from a cluster with $N$ atoms scales with $N^{5 / 3}$, the fraction of electrons which can escape drops significantly for larger clusters. Electrons that cannot overcome the Coulomb barrier lead to the formation of a nanoplasma, akin to inner ionization [7]. These quasi-free photoelectrons thermalize and the plasma core can recombine. The nanoplasma charge imbalance is neutralized by exploding off the outermost cluster layers. Such a Coulomb explosion of the cluster outer layers and recombination of the nanoplasma core is not limited to heterogeneous systems but is also expected to take place in larger homogeneous clusters. Experimentally, however, it is much more difficult to detect because the spatial origin of the charge states cannot be tracked with tof spectroscopy as in the presently investigated core-shell systems. Theoretically, cluster-core recombination has been suggested to explain differences in observed and calculated charge states [10]. Recent theoretical work predicts that such charge redistribution and neutralization processes will turn the cluster Coulomb explosion into a much slower (>ps) hydrodynamic expansion [26, 27]. 
In conclusion the fragmentation dynamics of pristine, doped and core-shell ArXe clusters irradiated by intense soft X-ray pulses at $13.7 \mathrm{~nm}$ and power densities exceeding $10^{14} \mathrm{~W} \mathrm{~cm}^{-2}$ have been investigated. Variation of the cluster composition allows tracking of the spatial origin and charge states of the fragments yielding insight into the nanoplasma dynamics. The lightly doped clusters exhibit an efficient charge transfer from the highly excited Xe dopants to the surrounding Ar matrix. The core-shell systems decay in a Coulomb explosion of the outer layers and recombination of the highly excited nanoplasma core with quasi-free electrons trapped in the cluster Coulomb potential. The core ion dynamics are better described by a delayed expansion rather than a Coulomb explosion. Such a delayed expansion of the core (or embedded bio-molecules) can be key for future single shot imaging experiments with x-ray lasers, improving the image quality and achievable resolution.

\section{Acknowledgments}

We would like to thank the DESY staff, in particular the Hasylab photon service team and the FLASH operators for their continuous support and Janos Hajdu for discussions. Funding is acknowledged from BMBF Grant No. 05KS4KTC1 and 05KS7KT1 as well as HGF Virtuelles Institut Grant No. VH-VI-103 and VH-VI-302.

\section{References}

[1] Kapteyn H C and Ditmire T 2002 Nature 420467

[2] Gaffney K J and Chapman H N 2007 Science 3161444

[3] Neutze R, Wouts R, van der Spoel D, Weckert E and Hajdu J 2000 Nature 406752
[4] Saalmann U and Rost J M 2002 Phys. Rev. Lett. 89143401

[5] Saalmann U, Siedschlag C and Rost J M 2006 J. Phys. B: At. Mol. Opt. Phys. 39 R39

[6] Hau-Riege S P, London R A, Chapman H N, Szoke A and Timneanu N 2007 Phys. Rev. Lett. 98198302

[7] Last I and Jortner J 2000 Phys. Rev. A 62013201

[8] Wabnitz H et al 2002 Nature 420482

[9] Santra R and Greene C H 2003 Phys. Rev. Lett. 91233401

[10] Siedschlag C and Rost J M 2004 Phys. Rev. Lett. 93043402

[11] Jungreuthmayer C, Ramunno L, Zhanghellini J and Brabec T 2005 J. Phys. B: At. Mol. Opt. Phys. 383029

[12] Georgescu I, Saalmann U and Rost J M 2007 Phys. Rev. A 76043203

[13] Bostedt C et al 2008 Phys. Rev. Lett. 100133401

[14] Chapman H N et al 2006 Nature Phys. 2839

[15] Ackermann W et al 2007 Nature Photon. 1336

[16] Sorokin A A, Bobashev S V, Feigl T, Tiedtke K, Wabnitz H and Richter M 2007 Phys. Rev. Lett. 99213002

[17] Hagena O F and Obert W 1972 J. Chem. Phys. 561793

[18] Wörmer J, Guzielski V, Stapelfeldt J and Möller T 1989 Chem. Phys. Lett. 159321

[19] Rutzen M, Kakar S, Rienecker C, von Pietrowski R and Möller T 1996 Z. Phys. D 3889

[20] Lengen M, Joppien M, Müller R, Wörmer J and Möller T 1992 Phys. Rev. Lett. 682362

[21] Tchaplyguine M, Lundwall M, Gisselbrecht M, Öhrwall G, Feifel R, Sorensen S, Svensson S, Martensson N and Bjorneholm O 2004 Phys. Rev. A 69031201

[22] Danylchenko O G, Doronin Y S, Kovalenko S I and Samovarov V N 2006 JETP Lett. 84324

[23] Danylchenko O G, Doronin Y S, Kovalenko S I, Libin M Y, Samovarov V N and Vakula V L 2007 Phys. Rev. A 76043202

[24] Haberland H, von Issendorff B, Kolar T, Kornmeier H, Ludewigt C and Risch A 1991 Phys. Rev. Lett. 673290

[25] Haberland H 1985 Surf. Sci. 156305

[26] Rusek M and Orlowski A 2005 Phys. Rev. A 71043202

[27] Ziaja B, Wabnitz H, Weckert E and Möller T 2008 New J. Phys. 10043003 